\title{
ESTADO DE LOS PROYECTOS AMBIENTALES ESCOLARES EN BOYACÁ
}

\author{
Aracely Burgos Ayala \\ Recibido el 22 de septiembre de 2015, aprobado el 14 de marzo de 2016 y actualizado \\ el 9 de diciembre de 2016
}

DOI: 10.17151/luaz.2017.44.4

\section{RESUMEN}

La Educación Ambiental (EA) se creó como una herramienta generadora de cultura ambiental. En Colombia, la EA formal se hizo realidad desde 1994 a través de la implementación de los Proyectos Ambientales Escolares (PRAE) en las Instituciones Educativas (IE). Actualmente se incrementan los daños a la naturaleza en Boyacá, pareciera que la cultura mencionada no existe; además, resulta escasa la información sobre la formulación y desarrollo de los PRAE como un punto de partida en la elaboración de programas y proyectos para el Plan Departamental de Educación Ambiental de Boyacá. Así, el objetivo fue analizar el estado de los PRAE de Boyacá, a través de: 1. Indicar sus generalidades, 2. Evidenciar características de su formulación; y 3. Indagar aspectos de su desarrollo. Para responder a estos objetivos se realizó una encuesta a $254 \mathrm{IE}$, obteniendo 175 respuestas. Se encontró que los PRAE se realizan prioritariamente en zonas urbanas; la mayoría tiene menos de cuatro años de creación, con una mínima asignación presupuestal. Así mismo, realizan una caracterización ambiental y una justificación, pero no reportaron los problemas del territorio de la zona de vida de las IE; priorizan temas como concientización, pero en las acciones persiste el manejo de residuos sólidos y el reciclaje, desconociendo otro tipo de conflictos de mayor impacto. La formulación y el desarrollo tuvieron escasa participación estudiantil y docente; su desarrollo no está articulado a los comités ambientales escolares, pero sí con otros actores. En consecuencia, resulta evidente la necesidad de un programa y proyectos específicos para la EA escolar en Boyacá, donde la institucionalidad asuma responsabilidad independiente y en conjunto frente al acompañamiento y participación tanto en formulación, desarrollo e impacto de los PRAE, con el fin de hacer efectiva la cultura ambiental acorde con los derechos de la naturaleza: respeto, responsabilidad y restauración.

\section{PALABRAS CLAVE}

Educación ambiental, cultura ambiental, instituciones educativas, Boyacá. 


\begin{abstract}
Environmental Education (EE) has been created as a tool of environmental culture. In Colombia, the EE became real in 1994 through of environmental school projects (ESP) in educational institutions (EI). Currently the damage to nature increases in Boyacá; no environmental culture, moreover, no detailed information exists about formulation and development of the ESP as one point of reference in the elaboration of programmes and projects for the Departmental Plan of Environmental Education, Boyacá. The aim was to analyze the state of Boyacá ESP through: 1. Indicate their generalities, 2. Evidence characteristics of its formulation; and 3. Investigate its development. It sent out a survey to $254 \mathrm{El}$ to gather data on these topics through of Departmental Education Secretary. Answered the survey in $175 \mathrm{El}$. It was found that: the ESP prevailing in urban areas; most have less than four years created, on a small budget. The formulation includes environmental characterization and justification, but not report problems in the Territory the life zone of schools; prioritize topics as awareness, however persists solid waste management and recycling. Formulation and development had student and teachers participation, the majority of natural sciences. Their development is not articulated with the School Environmental Committees, but with other actors. Therefore, it is clear that need for a program and specific projects in EE in Boyacá, where the institutions assumes independent and jointly liable to the accompaniment and participation both in formulation, development and impact of the ESP, in order to make effective of environmental culture consistent with the rights of nature: respect, responsibility and restoration.
\end{abstract}

KEY WORDS: environmental education, environmental culture, educational institutions, Boyacá.

\title{
INTRODUCCIÓN
}

La EA se pensó como una de las formas más factibles de crear una conciencia ciudadana, cuya finalidad fuera la conservación de la naturaleza (Vaughan et al., 2003; World Commission on Environment and Development. 1987), con una transformación de la realidad social (Macedo-Salgado, 2007), a través de la generación de procesos de prevención, reflexión, pensamiento crítico y toma de decisiones (Torres, 2010). En síntesis, su fin último es ser generadora de una cultura ambiental (Velasco, 2012), con cambios estructurales en lo individual y en lo colectivo. 
En Colombia, hace más de cuatro décadas, se inició la implementación de políticas para desarrollar la EA, las que han evolucionado y se han fortalecido (Burgos, 2013); sin embargo, sus resultados resultan imperceptibles. Esto hace pensar que si bien las políticas podrían ser las correctas y bien estructuradas, se desconoce el logro de sus objetivos (Badillo, 2012; Velásquez, 2010; Maldonado, 2005).

Ejemplo de ello son los PRAE que a pesar de ser establecidos desde el año 1994 a través de la Ley General de Educación, reglamentados el mismo año con el Decreto 1743,y reafirmados con la Política Nacional de Educación Ambiental (PNEA) del 2002, y con la Ley 1594 del 2012; a la fecha su impacto es desconocido.

Tales proyectos se plantearon como la forma más efectiva para desarrollar conciencia ambiental de los individuos en el contexto o zona de vida de la IE. Así se podía conservar y proteger la naturaleza, incluso, se podría pensar en nuevas formas o alternativas (Acosta, 2011, 2010). En este sentido, el reto del PRAE es ser un "proyecto de transformación del sistema educativo, reformulando el quehacer pedagógico y didáctico, elaborando modelos para la construcción del conocimiento y colaborando en la formación de nuevos valores y actitudes" (Holguín et al., 2010), a través del reconocimiento y aporte a las problemáticas ambientales, en el marco de diagnósticos participativos ambientales locales, regionales y nacionales, con miras a coadyuvar a la resolución de problemas ambientales específicos de la región donde se halla cada IE.

De forma particular en Boyacá, el Comité Interinstitucional de Educación Ambiental de Boyacá (CIDEABOY), en el proceso de formular los programas y proyectos del "Plan Departamental de Educación Ambiental de Boyacá (2015-2025)”, reconoció la escasez de información en cuanto a la EA formal en Boyacá, luego de más de veinte años de implementada la normatividad en Colombia, uno de los pocos departamentos que ha logrado avances en este sentido es Córdoba (Villadiego et al., 2011).

Sin pretender desconocer investigaciones como la de Pachón $(2011,2012)$, Vargas y Estupiñán (2012) y Pulido et al. (2014), o acciones como las de Corpoboyacá, quien acompañó a 434 IE en la incorporación de los PRAE al Plan Educativo Institucional (Corpoboyacá, Plan de Acción 2012-2015), la Secretaría de Educación de Boyacá y Holcim Colombia, esta última en el marco del desarrollo de la responsabilidad social empresarial, quienes coinciden en avances importantes y acompañamiento en varios PRAE del departamento; resulta evidente la necesidad de profundizar en aspectos como coherencia entre la realidad ambiental de las zonas de vida de las IE, así como el desarrollo de metodologías y pedagogías que permitan una correcta formulación y desarrollo de estos proyectos. 
Lo anterior toma relevancia al ser Boyacá un departamento rico en ecosistemas estratégicos como el páramo; tiene el 18.3 \% de los páramos de Colombia (Anaya y Echeverry, 2014) y bosques altoandinos, cuya biodiversidad ha sido bien documentada (Lagos et al., 2006; Cardozo et al., 2005; Aranguren et al., 2004; Márquez, 2003; Rangel, 2000), pero donde prevalece y se acentúa un alto índice de destrucción. Un ejemplo de ello es que entre el 2005 y el 2009 la transformación de los páramos fue de 21.3\% (Corso, 2013), dando a entender que, posiblemente, el conocimiento y apropiación de estos ecosistemas no ha permeado de forma contundente en las comunidades educativas que viven inmersas en ellos. Además, si bien estos cambios no corresponden particularmente a acciones escolares, es clara la necesidad de una cultura educativa ambiental, con el fin de que las nuevas generaciones crezcan con una mirada crítica y un sentido de pertenencia más profundo sobre su realidad ambiental.

Por lo tanto, en este artículo se presenta un análisis del estado de los PRAE de de Boyacá, sus generalidades, formulación y desarrollo. Generalidades tales como la ubicación -rural, urbana-, resultan relevantes al ser Boyacá un departamento rural casi en un $40 \%$ (DANE, 2005); igualmente, la temporalidad que tiene desde su formulación, así como el tiempo que llevan en desarrollo, así como la asignación presupuestal en los dos últimos años (2014-2014). La formulación y el desarrollo se logró según los lineamientos propuestos por Holguín et al., (2010). Por la complejidad metodológica no fue posible una evaluación del impacto de los PRAE, sin embargo, procedimientos como el uso de la matriz de vester se han usado para evaluar el conocimiento de los problemas así como las actitudes de las personas (Pulido et al., 2014).

\section{METODOLOGÍA}

La investigación se realizó en Boyacá (Figura 1), situado en el centro del país, sobre la cordillera oriental de los Andes; localizado entre los $04^{\circ} 39^{\prime} 10^{\prime \prime}$ y los $07^{\circ} 03^{\prime} 17^{\prime \prime}$ de latitud norte y los $71^{\circ} 57^{\prime} 49^{\prime \prime}$ y los $74^{\circ} 41^{\prime} 35^{\prime \prime}$ de longitud oeste. Cuenta con una superficie que representa el $2.03 \%$ del territorio nacional. 


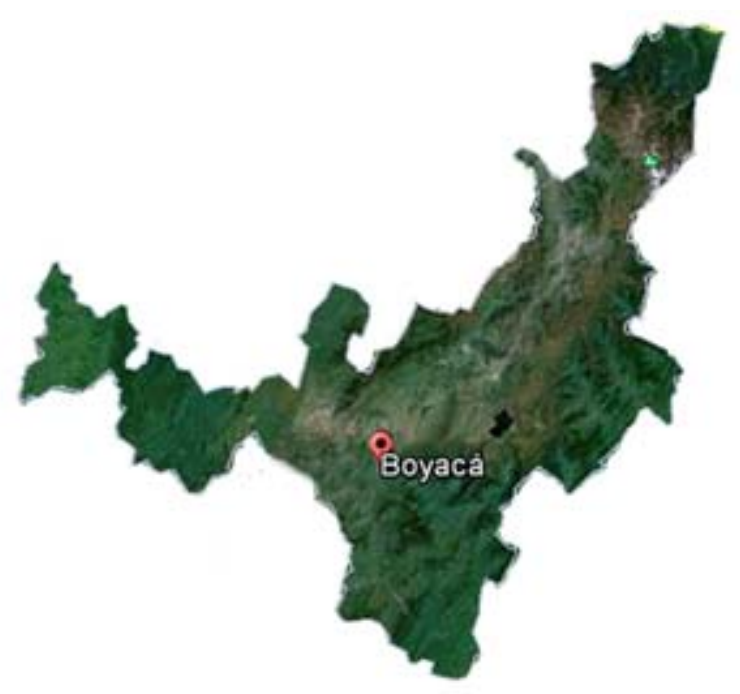

Figura 1: Mapa del departamento de Boyacá

Fuente: https://www.google.com/earth/

Dentro de las categorías de análisis estuvieron:

- Generalidades. De cada PRAE se revisó: ubicación de las IE (rural, urbana), fecha de creación e inicio, duración estimada, presupuesto asignado en los últimos años.

- Formulación. Se solicitó información que indica tanto el Decreto 1743, y de forma más específica, aspectos sugeridos por Holguín et al., (2010) en relación con: la descripción de la situación medio ambiental y social de la comunidad, descripción del por qué, el para qué y el cómo se llevará y se desarrolla el PRAE, así como la vinculación de entidades en la formulación del PRAE.

- Desarrollo. Se requirió información sobre: número total de estudiantes y docentes que hay en la IE y los que hacen parte del PRAE; área de los docentes que coordinan su desarrollo; existencia de un comité ambiental escolar que se articula al proyecto, y quiénes lo integran; su vinculación al proyecto educativo institucional (PEI) y actores que hacen parte de su desarrollo.

Como población se tuvo en cuenta a las 254 instituciones educativas de Boyacá en donde tiene injerencia la Secretaría Departamental de Educación, sin incluir las principales ciudades (Tunja, Duitama, Sogamoso, Chiquinquirá). Como muestra se tomaron 175 IE (69\% de total), puesto que fueron las que enviaron información dentro del tiempo estipulado, lo que garantizó que fuera una muestra al azar.

La información se obtuvo a través de la técnica de encuesta, como instrumento se usó el cuestionario con pregunta cerrada; este se construyó de tal forma que era claro lo que pretendía conocer sobre generalidades, formulación y desarrollo de cada PRAE. Se aclaró que al referirse a territorio o zona de vida se designaba a la región donde 
viven, que es dónde se halla la IE. Posteriormente, se realizó el proceso de tabulación y análisis de la información.

\section{RESULTADOS}

De forma general, se obtuvo una respuesta positiva desde las IE, pues de las 254 IE a quienes se les envió la encuesta, 175 respondieron oportunamente.

\section{Generalidades de los PRAE en Boyacá}

En cuanto al lugar donde se desarrolla el PRAE, se encontró que el $41 \%$ se desarrolla en zona urbana y el $37 \%$ en zona rural. Cerca del $68 \%$ se crearon después del año 2000 (Figura 2), lo que corresponde a la fecha de implementación; se resalta que hasta el año 2000, no eran más de veinte los PRAE creados e iniciados (y puestos en marcha).

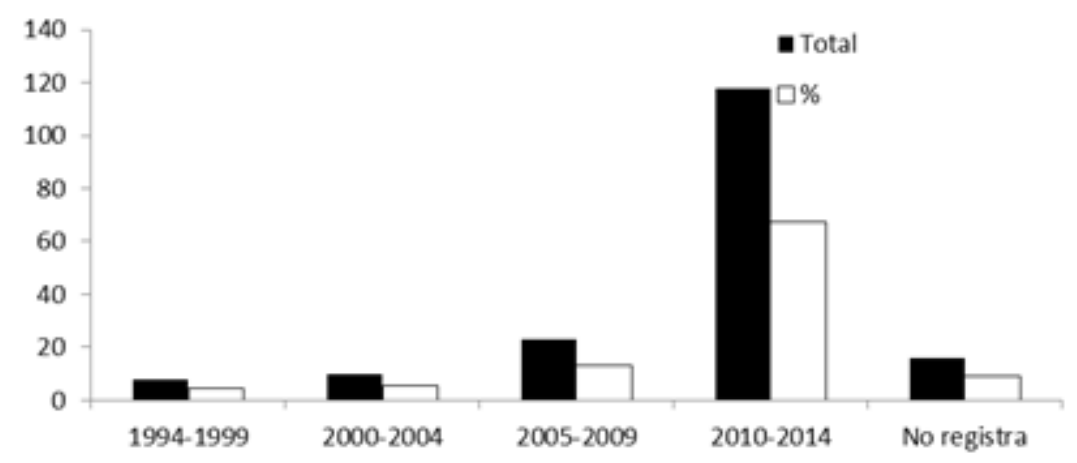

Figura 2. Fecha de creación de los PRAE de Boyacá

\section{Fuente: la autora}

Referente al presupuesto asignado a estos proyectos en los últimos dos años, se encontró que a $130 \mathrm{IE}(74 \%)$ no se les asignó ningún recurso; 24 contaron con 1 a 5 millones de pesos, dinero proveniente, en su mayoría, de aportes de las alcaldías.

\section{Características clave de la formulación de los PRAE en Boyacá}

El $58 \%$ de las IE tuvieron en cuenta la problemática ambiental de su territorio (T), mientras que el $25 \%$ tuvieron en cuenta la de la IE y tan solo el $11 \%$ (20 IE) tuvieron en cuenta al T. Sin embargo, del total de las IE, 140 (80\%) afirman haber realizado caracterización del territorio; similar resultado se encontró para la delimitación de la 
situación ambiental de la zona de vida de la IE y para el diagnóstico ambiental del T. Referente a la delimitación del problema, el $90 \%$ de las IE indican que sí realizaron delimitación y planteamiento del problema.

En cuanto al énfasis del PRAE, se encontró que estos proyectos pretenden trabajar predominantemente en temas como la concientización, el agua, el manejo de residuos sólidos y el reciclaje; en menor medida desarrollan los temas de fauna, paisaje y ecología humana (Figura 3).

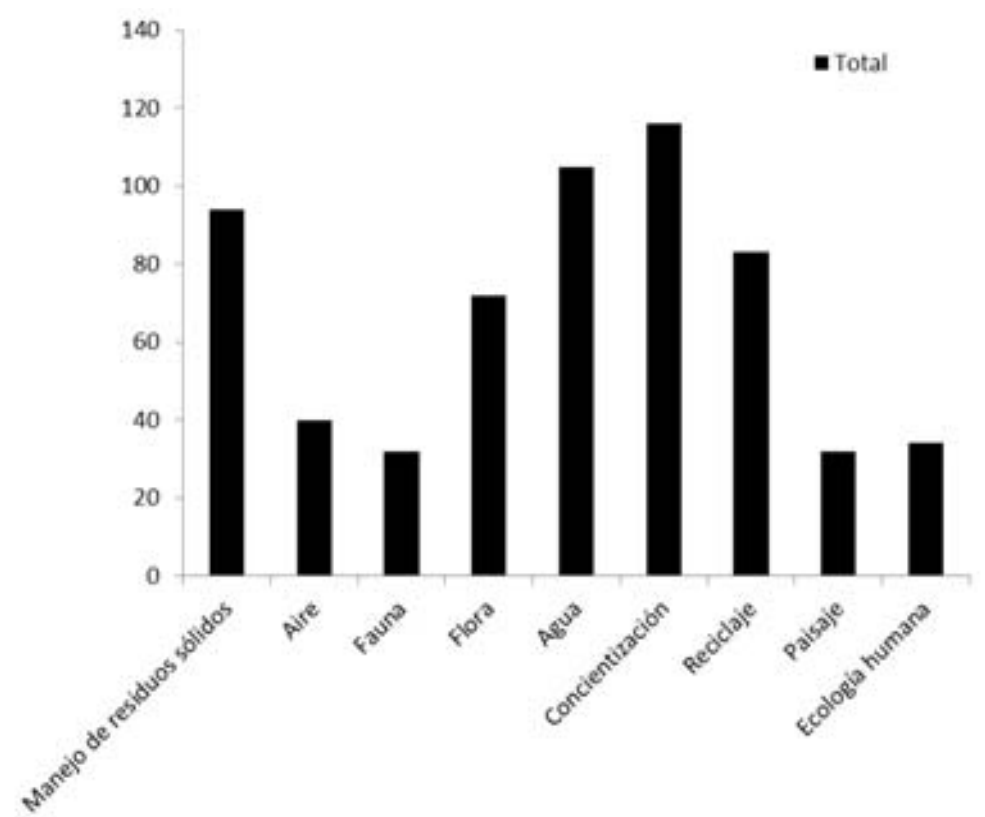

Figura 3. Ėnfasis de los PRAE de las IE de Boyacá. Fuente: la autora

El 95\% de las IE (167) indicaron haber realizado una justificación y objetivos generales y específicos del PRAE al momento de formularlo. Un total de 44 PRAE $(25 \%)$ no incluyó metas, y solo el 57\% contemplan indicadores; asimismo, 68 IE (39\%) indicó fuentes de verificación. En cuanto a la inclusión de cronograma de actividades, el 93\% (163 del total de las IE) respondieron que sí lo contenían.

También se indagó por la participación de los docentes y estudiantes en la construcción del PRAE; frente a esto, se encontró que en el $90 \%$ de las IE sí participaron los docentes, similar fue el resultado para estudiantes.

\section{Aspectos del desarrollo del PRAE}

Se indagó si a través del PRAE se invitaba a la comunidad educativa (CE) a ser parte activa de la solución a los problemas ambientales de la IE, específicamente se 
preguntó por la participación de los estudiantes y de los docentes en el PRAE. Estableciendo rangos de porcentajes, se encontró que 77 IE articulan entre el 76 y el $100 \%$ de sus estudiantes, y solo 44 IE articulan entre el 0 y el $25 \%$. Sin embargo, al preguntar los grados de los estudiantes que participan el PRAE, se halló que solo el $49 \%$ de IE vinculan a niños de todos los grados, seguido por IE que vinculan exclusivamente a estudiantes de básica secundaria y media (34\%) (Figura 4).

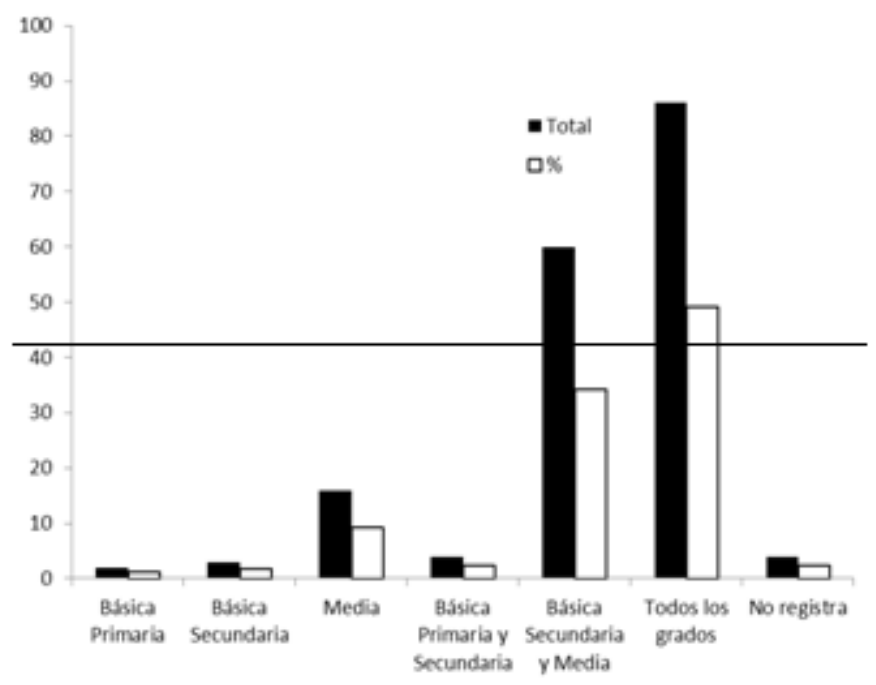

Figura 4. Grado de los estudiantes que participan en el desarrollo del PRAE de las IE de Boyacá. Fuente: la autora

Contrario a los estudiantes, 73 IE vincularon del 0 al $25 \%$ de sus docentes al PRAE, seguida por $50 \mathrm{IE}$, que afirman vincular entre 76 y el $100 \%$. Adicionalmente, las áreas de los docentes que participan son mayoritariamente de ciencias naturales y afines (44\%) (biología, agrarias, agropecuarias); además, se encontró que solo el $31 \%$ de las IE vinculan a docentes todas las áreas, y en un $24 \%$ vinculan a docentes de otras como matemáticas, castellano, inglés, química y otros (Figura 5). 


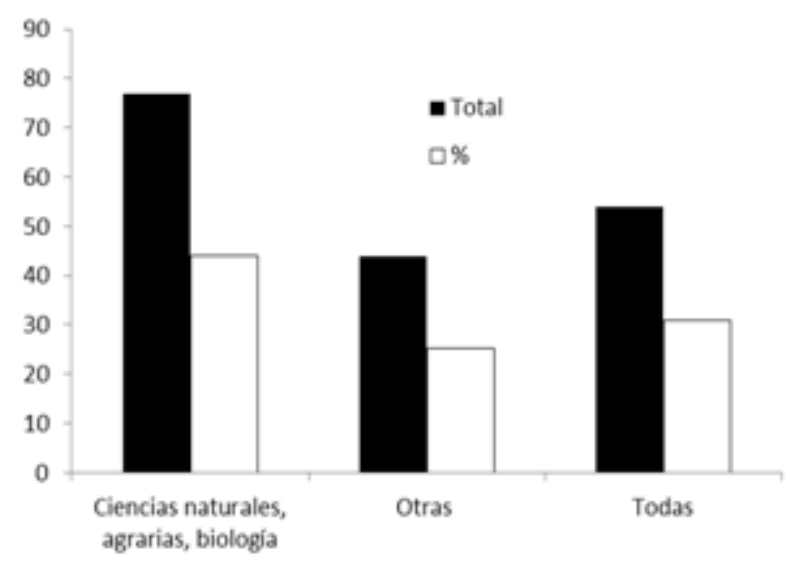

Figura 5. Areas de los docentes que se integran en los PRAE de las IE de Boyacá. Fuente: la autora

Articulado a la participación de la CE en el PRAE, se indagó si existía un comité ambiental escolar (CAE), donde se encontró que el $43 \%$ de las IE (76) no lo tenía. Otra forma de verificación de la participación de toda la IE en los PRAE, fue a través de la articulación de este proyecto con el PEI; al respecto se halló que el $11 \%$ de las IE (19) no tienen incluido el PRAE dentro del PEI.

Respecto a otros actores que estaban involucrados en el desarrollo del PRAE, se mencionaron algunos como: las Unidades Municipales de Asistencia Técnica Agropecuaria, Corpoboyacá, alcaldías, empresas de servicios públicos, Secretaría de Educación, la Corporación Autónoma Regional, padres de familia, Corpochivor, Cruz Roja, ESE Salud, Universidad Pedagógica y Tecnológica de Colombia (UPTC), Fundación Universitaria Juan de Castellanos, Universidad Santo Tomás, Proyecto Ondas, Holcim Colombia, Comités Técnicos Interinistitucionales de Educación Ambiental (CIDEA) municipales, el SENA, CorpoOrinoquía, ONG Planeta Vivo, Parques Nacionales Naturales, Fundación Ecohumana, Juntas de Acción Comunal, Centro Juvenil Campesino, Red juntos, Epsagro, Cabildo verde, Policía Nacional, comités ambientales, entre otros. Se nombran con mayor frecuencia a la UPTC, a las corporaciones y alcaldías.

\section{DISCUSIÓN}

\section{Generalidades de los PRAE en Boyacá}

En vista que se encontró un predominio del desarrollo de PRAES en la zona urbana resulta preocupante, puesto que la población de Boyacá es rural en un 49\% (DANE, 
2005), y se halla en vulnerabilidad por razones como la escasez de los beneficios que provee la naturaleza, y la destrucción de la misma, de la cual, a su vez, depende su economía (Forero, 2002). Frente a esto, se descarta el sesgo posible por reporte de PRAE en las IE de las ciudades principales, pues estas se excluyeron de la investigación.

Aunado a lo anterior, el hecho que Boyacá sea rural y que sus ecosistemas como los páramos y los bosques altoandinos se han identificado como frágiles por diversas problemáticas agudizadas en las últimas décadas, le imprime importancia a aspectos como la fecha de la creación e inicio del PRAE. Entonces, conviene cuestionarse por qué luego de 20 años de institucionalizados estos proyectos escolares en las IE de Colombia, lleven, en su mayoría, tan solo 4 años de creados en Boyacá; esto se corrobora con lo hallado en otros estudios (Pulido et al., 2014; Camargo y Chávez, 2010), y evidencia que tan solo pareciera ser un requisito formal ante las secretarías de educación, pero no se denota un compromiso frente a la importancia de mismo.

Una de las explicaciones posibles es la falta de claridad en los conceptos, en la contextualización de la realidad local, regional y global, la dificultad para el abordaje pedagógico y en la comprensión de lo que es un problema ambiental (Pérez y Porras, 2011), así como la ausencia de claridad en los lineamientos para su formulación (Holguín et al., 2010). Las implicaciones más profundas serían la reducida contribución a la formación de nuevas generaciones de ciudadanos conscientes, con aptitud, conocimiento, motivación y compromiso para trabajar individual y colectivamente hacia la solución y prevención de los problemas ambientales (Decreto 1743, 1994; Ley 115,1994; Tbilisi, 1977), a través de la participación inmediata de la CE, y a futuro, del establecimiento de una verdadera cultura ambiental.

Lo anterior también podría justificarse por la tendencia a la baja y casi nula asignación presupuestal para la ejecución de estos proyectos. En este punto cabe cuestionarse: ¿a quién le corresponde velar formalmente por la cultura en educación ambiental? Por lo tanto, revisar si cada IE y actor, en general, está asumiendo su compromiso. Lo anterior, sin ignorar los apoyos brindados por entidades como Holcim Colombia, Corpoboyacá y Corpochivor, y algunas alcaldías, quienes han invertido en algunos PRAE, pero resulta mínimo su esfuerzo para lo que se requiere en el departamento.

\section{Características clave de la formulación de los PRAE en Boyacá}

Varios aspectos se deben tener en cuenta en la formulación correcta de un PRAE; aquí se consideran algunos de relevancia, como la delimitación de la problemática ambiental de la zona de vida o T donde se encuentra cada IE, por tanto del problema, la caracterización y situación ambiental general y la justificación. La mayoría de las IE afirma haber realizado caracterización ambiental, lo que es la base para una adecuada 
delimitación de la situación ambiental; sin embargo, cerca de la mitad de las IE tuvieron en cuenta la problemática de la zona de vida o del T que la rodea. En este sentido, podría esperarse que la delimitación y el planteamiento del problema formulado en el PRAE no contengan la realidad de la necesidad ambiental. Las implicaciones de esta situación convergen en que todo lo que derive de un inadecuado diagnóstico, terminará en el mismo sentido.

Así, resulta importante que todo PRAE se fundamente no solo en unas bases conceptuales y filosóficas, sino que provenga de un proceso reflexivo a partir del diálogo de diversas disciplinas (Camargo y Chávez, 2010), y de la realidad ambiental de cada T e IE, para evitar "la raíz de una pobreza ecológica" (Jackson, 2000) por la ausencia de un planteamiento que además cuente con enfoque sistémico (Serna, 2007).

Lo mencionado podría contrastarse cuando se evidencia el énfasis de los PRAE con la realidad ambiental del departamento. Resulta favorable que el tema privilegiado fue la concientización y el agua, perfectamente coherente con la intencionalidad de estos proyectos escolares (Decreto 1743, 1994; Ley 1549 del 2012), y en general de la EA. Sin embargo, se aprecia que los temas siguientes, y según su grado de importancia, son el manejo de residuos sólidos y el reciclaje; esto implica, posiblemente, que no se realizó una revisión minuciosa de la situación y problemática ambiental tanto de la IE como del T, puesto que el departamento tiene aspectos relevantes para desarrollar en la instancia de EA en lo formal, como lo son la pérdida de ecosistemas estratégicos, recuperación de afluentes hídricos, flora y fauna, entre otros, que competen a prácticamente todas las zonas de vida donde se hallan las IE.

Adicionalmente, aunque prácticamente todos afirman contar con una justificación, podría pensarse que ésta no cuenta con lo que realmente requiere la naturaleza cercana a su IE; en suma, quedarían sin fundamento ambiental en cuanto al por qué, para qué y cómo se proyecta el PRAE.

La ambigüedad planteada implica la necesidad de revisar a detalle los documentos de formulación del PRAE, pues se logró evidenciar por Pulido et al., (2014) y a través de cuatro documentos de formulación PRAE, que no se cuenta con la justificación de estos proyectos acorde con la realidad ambiental de cada municipio y zona de vida de las IE.

Adicionalmente, una correcta formulación de cualquier proyecto debe apreciar metas, indicadores y cronograma; sin embargo, la cuarta parte de los PRAE de las IE de Boyacá no tiene metas y casi la mitad no cuenta con indicadores, lo que implica que se desconoce cómo se van a lograr y a medir los avances, sin lo cual queda imposible hacer mejoras y ajustes a futuro. Por el contrario, casi la totalidad indican tener un 
cronograma definido, pero las actividades posiblemente no estén permitiendo el logro de los objetivos, tanto del PRAE como de la EA (Bermúdez y DeLonghi, 2012, Torres, 2010).

En cuanto a la participación de docentes y estudiantes, de una parte,se indica que gran porcentaje de estudiantes y docentes hizo parte de la formulación; sin embargo, esta respuesta es cuestionable, puesto que la investigación de Pulido et al., (2014) evidencia que el proyecto fue construido solamente con un docente y el rector, lo que sería otro obstáculo para el éxito del PRAE. El ideal es contar con un diagnóstico ambiental participativo de toda la CE (estudiantes, docentes, personal administrativo, directivos, familia, vecinos), que visibilicen experiencias ambientales previas (Pérez y Porras, 2011; Camargo y Chávez, 2010; Holguín et al., 2010). Asimismo, la formulación requiere acompañamiento o asesorías de otras instituciones, por ejemplo las de educación superior, estrategia que ha funcionado en otras IE (Pachón, 2012; Guerra, 2002; Santoyo, 1998).

\section{Aspectos del desarrollo del PRAE}

El desarrollo del PRAE, al igual que su formulación, requiere de la participación plena de todos los actores de la CE. Para las IE de Boyacá, la vinculación estudiantil al PRAE estuvo poco representada, contrario a lo que debería ser; si bien la mayoría de las IE involucran a estudiantes de todos los grados, varias incluyen solo a los de básica secundaria y media. En consecuencia, se esperaría que sean pocos los niños que logren hacer realidad en su vida una EA real, por tanto, una vez más se cuestiona la cultura ambiental promovida desde los PRAE en Boyacá.

La participación de la población docente en el desarrollo del PRAE en las IE en Boyacá fue negativa. La mayoría de IE tan solo vinculan hasta el $25 \%$ del total de docentes, lo que se complejiza al ser más representativos los docentes de las áreas de ciencias naturales, biología y agrarias. Sus implicaciones son la escasa interdisciplinariedad y transversalidad del PRAE dentro del currículo.

Al respecto, Hungerford et al. (1994), señalan una propuesta de currículo de EA escolarizada que incluya conocimientos conceptuales del ambiente y de la sociedad y su interrelación, que permitan reconocer las problemáticas y posterior, hacer planteamientos y acciones. Fortier et al., (1998), propone el siguiente orden en el currículo: preguntas, análisis, conocimiento de procesos y sistemas ambientales, habilidades de investigación, toma de decisiones y acción, para finalizar en una responsabilidad personal y cívica. Este tipo de guías integraría el PRAE, el PEI y el currículo, con interdisciplinariedad de asignaturas y la integración de la CE, y no solo un grupo determinado de estudiantes y docentes, estos últimos que además, puede que no cuenten con la capacitación para tal fin (Torres, 2010). 
Afortunadamente, la mayoría de las IE afirman que sí articulan el PRAE con el PEI; lo preocupante es que no se evidencia su impacto, debido, posiblemente, a que la mayoría de los PRAE son muy recientes y a la baja participación de docentes y estudiantes. Los efectos del distanciamiento entre el PEI y el PRAE, si es que así fuera, sería la carencia de procesos pedagógicos (Pachón, 2012; Maldonado, 2005), baja integración del PRAE en todas las sedes de la IE (Pachón, 2012), y poca capacitación a todo el personal docente sobre la cultura ambiental (PNEA, 2012).

De otra parte, la participación de la CE en los PRAE de Boyacá también se apreció limitada en lo referente a la existencia de los CAE. De esta forma, resultan pocos los actores que propiamente se articulan con el proceso educativo ambiental. Lo anterior se reafirma frente a la PEA (2012), la que sugiere que todos los actores del sistema educativo tengan espacio de diálogo y participación, preferiblemente, por motivación personal en su identidad con el ambiente en el que vive. Al respecto, una investigación particular indica que el PRAE sí invita a toda la CE a ser parte activa del proceso ambiental de la IE, pero las actividades propuestas y realizadas en la metodología no reflejan el interés del PRAE ni de la EA (Pulido et al., 2014).

A pesar de lo mencionado, resulta importante que la mayoría de las IE nombran una diversidad de actores involucrados en el desarrollo del PRAE, cuya participación juega un rol importante en cuanto a apoyar la formulación y desarrollo del PRAE, así como el acompañamiento en la búsqueda del logro sus objetivos; lo contrario sería una actividad poco participativa (Torres, 2011; Camargo y Chávez, 2010). Por ejemplo, desde la formulación, la gama de problemas indicados por todos los actores de la CE aportarían significativamente al diagnóstico ambiental; de esta forma, se tendría una lectura complementaria y crítica de contexto, por tanto, un punto de partida fundamental para el reconocimiento de las dinámicas naturales y socioculturales en las que se dinamiza la problemática, la construcción de referentes asociados a las formas de relación de los actores y los escenarios desde los cuales es factible actuar en la búsqueda de soluciones pertinentes, con base en la praxis de la ecología social que orienta a las futuras generaciones en su interacción con el ambiente (Gudinas y Evia, 1995).

Al respecto, las universidades actuarían en la formulación, el desarrollo y el impacto. Instituciones como el Estado (por ejemplo, alcaldías y diversas secretarías), deberían acompañar en la asignación de tiempos y recursos a cada proyecto, máxime en su compromiso educativo ambiental hacia el logro de nuevas generaciones de ciudadanos. Las corporaciones autónomas regionales, por su parte, tienen la función de hacer veeduría del desarrollo de los diferentes aspectos de los PRAE, así como su articulación con los comités municipales de educación ambiental. Igualmente, resulta clave la participación activa de organizaciones privadas y de la sociedad civil del T 
donde se encuentra cada IE, para aunar esfuerzos y converger en un verdadero cambio cultural ambiental.

Finalmente, si bien esta investigación no involucró conocer el impacto del PRAE en Boyacá, es importante reconocer la importancia de una congruencia entre los problemas ambientales prioritarios de la zona de vida de cada IE, según la participación y aporte de cada actor de la CE y las actuaciones de esta respecto a los problemas, con un resultado debería ser un impacto positivo. Se han evidenciado inconsistencias entre la realidad ambiental de los territorios y lo que perciben, por ejemplo, los estudiantes y los docentes (Pulido et al., 2014). Dicha situación cuestiona el proceso educativo ambiental adelantado por las IE, el cual debe facilitar procesos para reconocer el entorno ambiental local desde las aproximaciones conceptuales y proyectivas (Pérez y Porras, 2011; Ardila, 2007), asunto que es una deuda consolidar, pero que por lo revisado se podría predecir que no se proyecte positivo.

\section{CONCLUSIONES}

En cuanto a las generalidades, se encontró que los PRAE de las IE de Boyacá se realizan prioritariamente en zonas urbanas, lo que no concuerda con que la mitad de la población de Boyacá vive en zonas rurales, que son además las que tienen mayores problemas ambientales.

La mayoría de estos proyectos se han formulado en los últimos 4 años, aun cuando se implementaron hace más de 20 , lo que indica que han sido tan solo una formalidad, pero sin un impacto fuerte en una cultura ambiental ciudadana. Dentro de las posibles explicaciones, una podría estar en la asignación presupuestal que resultó prácticamente nula, por tanto cuestiona a los actores que tienen esta responsabilidad. Además, si bien los PRAE cuentan con una caracterización de la situación ambiental y la justificación, pocas revisaron en detalle lo que ocurría en el territorio y las zonas de vida de las IE, por ende, la delimitación de la problemática ambiental y el planteamiento del problema no resultan acordes con la realidad, razón por la cual se cuestiona el cómo se espera que resulte todo lo que se derive de ello. Al respecto, resultó importante que la concientización fue el tema de mayor interés, consecuente con la política de la EA, sin embargo persisten los temas como manejo de residuos sólidos y reciclaje, no concordantes con la generalidad de la problemática ambiental de Boyacá. Adicionalmente, la formulación de los PRAE de las IE de Boyacá no tiene metas e indicadores claros, por tanto, resulta complejo hacer seguimiento y ajustes, 
que podrían estar incluidos en el cronograma de actividades, los que sí aparecen en la mayoría de PRAE de las IE.

De otra parte, tanto la formulación como el desarrollo de los PRAE contaron con la participación de docentes y estudiantes, sin embargo, su representación resultó escasa. La participación docente, además de ser limitada, se restringía en su mayoría a áreas relacionadas con las ciencias naturales, por ende, con una consecuente baja transversalidad y participación en el currículo, contradictorio con la afirmación desde las IE, de que estos proyectos sí están articulados a los PEI. En cuanto a los estudiantes, no se vincula a toda la población, lo que podría estar dando cuenta de un mínimo impacto del propósito último de la EA en este nivel de formación, y en general, de consolidar una nueva generación de ciudadanos con una fuerte cultura ambiental.

En el desarrollo también se encontró una limitada articulación con los comités ambientales escolares, sin embargo se aprecian incoherencias, pues lo reportado por las IE y la literatura consultada, reporta el acompañamiento de diversos actores en su formulación y desarrollo.

Finalmente, esta investigación evidencia la necesidad de un programa y proyectos específicos para la educación ambiental escolar en Boyacá, donde toda la institucionalidad asuma su responsabilidad independiente y en conjunto frente al acompañamiento y participación directa o indirecta, no solo a la formulación, el desarrollo y al impacto de los PRAE, sino con la intención de poder visualizar a futuro cercano y lejano la naturaleza del departamento, verdaderamente acorde con el respeto, la responsabilidad y la restauración, prácticas socioculturales propuestas desde el buen vivir necesarias de realizar en toda institución educativa.

\section{AGRADECIMIENTOS}

La autora expresa sus agradecimientos a Julián Pulido Rojas y a Alexander García Rodríguez, trabajadores sociales, por su colaboración en la tabulación de los datos. Igualmente a Blanca Ibáñez Cruz, funcionaria de la Secretaría de Educación de Boyacá. Finalmente, expreso agradecimientos a todos los integrantes del Comité Técnico Interinstitucional de Educación Ambiental de Boyacá (CIDEABOY), por la oportunidad de realizar esta investigación. 


\section{REFERENCIAS}

- Acosta, A. (2011). Los derechos de la naturaleza: una lectura sobre el derecho a la existencia. En: Acosta, A. y Martínez, E. (2011). La naturaleza con derechos, de la filosofía a la política. Ediciones Abya-Yala. Quito, Ecuador. 376p.

- Acosta, A. (2010). El buen vivir, una utopía por (re)construir. En: Sempere, J., Acosta, A., Abdallah, S y Ortí, M. (2010). Enfoques sobre bienestar y buen vivir. Centro de investigación para la paz (CIP-Ecosocial). Madrid. 56p.

- Anaya, J. y Echeverry, E. (2014). Extractivismo en Boyacá y nuevas oportunidades de movilización social. Recuperado el 15 de noviembre del 2014 de www.cinep.org.co

- Aranguren, N., Monroy, D. y Gaviria, S. (2007). Los crustáceos planctónicos del Lago de Tota (Boyacá, Colombia). Ciencia en Desarrollo, 1(2): 64-81.

- Ardila, J. (2007). Intervención de trabajo social en la dinamización de los proyectos ambientales escolares (PRAES) de la Institución Educativa Departamental Técnico Agropecuaria San Ramón del municipio de Funza con los estudiantes de grado once mediante la aplicación de la metodología de desarrollo empresarial participativo (DEP), desde el consultorio social Uniminuto agosto 2006 a junio 2007. Disponible en. http://repository.uniminuto.edu:8080/jspui/bitstream/10656/

- 112/1/TTS_ArdilaGomezJuliePauline_2007.pdf

- Badillo, M. 2012. Política de educación ambiental en Colombia, 2002-2010. Revista de investigación agraria y ambiental. 3 (1): 89-96.

- Bermúdez, G. y De Longhi, A. (2012). El conocimiento didáctico de contenidos biológicos de ecología. En: Flores, C. Experiencias latinoamericanas en educación ambiental. Editorial CECyTE NL-CAEIP, Monterrey, México.

- Burgos, A. 2013. La educación ambiental: impacto y conocimiento de sus fines y formas. Cultura Científica, 11, 20-31.

- Camargo, C. y Chávez, A. (2010). Propuesta de apoyo para la formulación del PRAE con énfasis en el plan de gestión de residuos sólidos. Estudio de caso en el Colegio Nuestra Señora de la Sabiduría (Bogotá). Revista Gestión Integral en Ingeniería Neogranadina, 2 (1).

- Cardozo, A., Bermúdez, A., Aranguren, N. y Duque, S. (2005). Algas Planctónicas del Lago de Tota: Listado Actualizado. Ciencia en Desarrollo, 2 (1): 44-58. 
- Corpoboyacá, Plan de Acción 2012-2015.

- Corso, G. (2013). Una mirada desde los páramos a la conservación de la biodiversidad en Colombia. En Cortés-Duque, J. \& Sarmiento, C. (Eds). (2013). Visión socioecosistémica de los páramos y la alta montaña colombiana: memorias del proceso de definición de criterios para la delimitación de páramos. Instituto de Investigación de Recursos Biológicos Alexander von Humboldt. Bogotá, D.C. Colombia.

- DANE - Departamento Administrativo Nacional de Estadística (2005).

- Decreto 1743 (1994). Por el cual se instituye el Proyecto de Educación Ambiental para todos los niveles de educación (...). DO 41476

- Forero, J. (2002). Economía campesina, estructura agraria y sistema alimentario. En J.M. Blanquier y C. Gros (Editores): Las dos Colombias. Editorial Norma.Bogotá.

- Fortier, J., Grady, S., LEE, S. y Marinac, P. (1998). Winsconsin's Model Academic Standards for Environmental Education. Publication Sales Wisconsin Department of Public Instruction. Bulletin No. 9001.

- Guerra, M. (2002). Incorporación de la temática ambiental en la educación formal básica rural y urbana del municipio de Suesca. Tesis Pontificia Universidad Javeriana.

- Gudynas, E. y Evia, G. (1995). Ecología social, Manual de metodologías para educadores populares. España. Editorial popular, S.A; Madrid.

- Holguín, M., Bonilla, P., Pupu, A., Lezaca, J. Rodríguez, I. \& Rodríguez, T. (2010). Guía metodológica para la formulación de proyectos ambientales escolares. Un reto más allá de la escuela. Impresión: Panamericana Formas e Impresos S.A.

- Hungerford, H., Volk, T. y Ramsey, J. (1994). A prototype environmental education curriculum for the middle school. UNESCO-UNEP International Environmental Education Programme.

- Jackson, M. (2000). A Future for the Indian Village. Asian Agri-History, 4(2), 105-124.

- Ley 115 de 1994. Por la cual se expide la Ley General de Educación. Febrero 8 de 1994, DO 41.214

- Ley 1549 del 2012. Por medio de la cual se fortalece la institucionalización de la política nacional de educación ambiental y su incorporación efectiva en el desarrollo territorial. Julio 5 del 2012. DO 48.482 
- Lagos, M., Zabala, J., Acosta, N. y Sánchez, C. (2006). "Dicotiledóneas" Flórula De La Reserva Forestal Protectora "El Malmo" Tunja (Boyacá-Colombia) ISBN: 978 - 958 660 - 115-3, Ed: Universidad Pedagógica y Tecnológica de Colombia, p. 63-81.

- Macedo, B. y Salgado, C. (2007). Educación ambiental y educación para el desarrollo sostenible en América Latina. Forum de Sostenibilidad, Cátedra de la UNESCO, 29-37.

- Maldonado, H. 2005. La educación ambiental como herramienta social. Revista: Geoenseñanza. 10 (1): 61-67.

- Márquez, G. 2003. Ecosistemas estratégicos de Colombia. Universidad Nacional de Colombia $15 p$.

- Pachón, N. (2011). La Educación Ambiental (EA) en la escuela: avances y dificultades de los PRAE del área de jurisdicción de Corpochivor. IV Congreso Internacional de Pedagogía, Currículo y Didácticas. Universidad Pedagógica y Tecnológica de Pereira.

- Pachón, N. (2012). Educación Ambiental (EA) en la Institución Educativa Integrada de Combita, Boyacá - Colombia: diagnóstico preliminar. V Congreso Internacional por el Desarrollo Sostenible y el Medio Ambiente. Universidad de Manizales.

- Pérez, M. y Porras, Y. (2007). Estudio para la identificación de tendencias en educación ambiental en instituciones educativas del distrito capital. Nodos y Nudos. 3 (22): 71-79.

- Política Nacional de Educación Ambiental, PNEA (2002). Ministerio de Medio Ambiente y Ministerio Nacional de Educación. Disponible en:http://cmap.upb.edu.co/rid=1195259861703_152904399_919/

- politia_educacion_amb.pdf Consultado el 14 de junio del 2014.

- Pulido, J., García, A. y Burgos, A. (2014). Formulación, Desarrollo e impacto del proyecto ambiental en la Institución Educativa Juan José Neira, Gachantivá, Boyacá. Tesis de pregrado, Fundación Universitaria Juan de Castellanos.

- Plan Departamental de Educación Ambiental de Boyacá (2014). Comité Interistitucional de Educación Ambiental de Boyacá. Sin publicar.

- Rangel, O. (2000). La región paramuna y franja aledaña en Colombia. Colombia Diversidad Biótica III La región de vida paramuna. Universidad Nacional de ColombiaInstituto de Ciencias Naturales, Instituto de Investigación en Recursos Biológicos Alexander von Humboldt. 
- Santoyo, M. (1998). Estudio exploratorio sobre la incorporación de la dimensión ambiental en los colegios de las Hermanas Bethlemitas. Tesis Pontificia Universidad Javeriana

- Serna, A. (2007). El derecho a un ambiente sano y la pedagogía ambiental. AGO.US. 7 (2), 345-359

- Tbilisi (1977). Intergovernmental Conference on Environmental Education organlied. Unesco (14-16 de Octubre).

- Torres, N. (2010). Las cuestiones sociocientíficas: una alternativa de educación para la sostenibilidad. Revista Luna Azul, 32, 45-52

- Vargas, C. y Estupiñán, M. (2012). Estrategias para la educación ambiental con escolares pobladores del páramo Rabanal (Boyacá). Luna Azul, 34; 10.25.

- Vaughan, C, Gack, J. Soloranzo, H. y Ray, R, (2003). The effect of environmental education on school children, their parents, and community members: a study of intergenerational and intercommunity learning. Journal Environmental Education, 34, $12-21$.

- Velasco, J. (2012). Cultura y educación ambiental: una mirada desde la antropología social. En: Flores, C. Experiencias latinoamericanas en educación ambiental. Editorial CECyTE NL-CAEIP, Monterrey, México.

- Velásquez, M. (2010). Evolución de la política nacional ambiental en materia de desarrollo urbano sostenible. En: Diez años de investigación jurídica y sociojurídica en Colombia: Balances desde la red sociojurídica; Londoño, B. y Gómez, D. Editoras. Editorial: Universidad Colegio Mayor de Nuestra Señora del Rosario, Tomo II: 66-105.

- Villadiego, J., Cardona, C., Ortiz, R., Coneo, M. y Ramos, A. La educación ambiental en el departamento de Córdoba: El caso de los proyectos ambientales escolares PRAE. Revista de Didáctica Ambiental, 7 (10): 7-15.

- World Commission on Environment and Development. 1987. Our Common Future. Disponible en: http://www.un-documents.net/our-common-future.pdf. Consultado el 23 de Julio del 2015. 
1. Magíster en Ciencias Biológicas, Universidad de Chile. Docente-Investigadora, Centro de Investigación, Facultad de Educación, Fundación Universitaria Juan de Castellanos. Integrante del Comité Técnico Interinstitucional de Educación Ambiental de Boyacá. Correo electrónico: burgos.aracely@gmail.com. orcid.org/0000-0002-1799-5791

Para citar este artículo: Burgos-Ayala, A. (2017). Estado de los proyectos ambientales escolares en Boyacá. Luna Azul, 44, 39-58. DOI: 10.17151/luaz.2017.44.4.

Recuperado de http://200.21.104.25/lunazul/index.php?option=com_content\&view=article\&id=219

Esta obra está bajo una Licencia de Creative Commons Reconocimiento CC BY

$$
\text { (9) } 1
$$

